\title{
THE DEVELOPMENT AND DESIGN OF THE \\ .SURGICAL SCHEDULER'S \\ HOSPITAL MANAGEMENT GAME \\ USING GASP II
}

Hoffman, Richard 0., Department of Industrial and Management Systems

Engineering, University of Nebraska, Lincoln, Nebraska and Paul E.

Torgersen, Dean of Engineering, Virginia Polytechnic Institute and

State University, Blacksburg, Virginia.

\section{ABSTRACT}

This paper presents a game designed to introduce the participants to the scheduling function of a hospital's surgical service. The participant is responsible for scheduling a set of operations each period. For each set of operations that have been scheduled, the participant receives a performance and financial report, including a measure of scheduling effectiveness, hospital and social costs, and a comparison of the actual schedule with the best possible schedule for that day. The game is set within a probabilistic environment, includes an adaptive learning feature, and uses GASP II simulation language.

\section{INTRODUCTION TO THE PROBLEM}

In 1969, Americans spent 60.3 billion dollars on health care and this amounted to 6.7 per cent of the gross national product. On a per person basis, the cost of health care was 293 dollars - more than twice the amount spent in 1959. In the past several years, health care costs have been rising at an average annual rate of 7 per cent. As a result, many people no longer can afford health care. The rise in hospital costs has also resulted in an increase in the cost of health insurance, which is now too expensive for many families to purchase. 2

A number of proposals have been suggested to solve, or at least ameliorate, what has been described as a national health crisis. These proposals include national health insurance, rate regulations, franchise health care, and management training. Emphasis has aiso been focused upon increasing the educational levels of hospital employees. As a result, the American Society for Hospital Education and Training of the American Hospital Association has been established for the purpose of improving the methods of training and the education of hospital personnel. This emphas is has been necessary because hospitals have traditionally hired non-professional employees with marginal qualifications and then paid them low wages. These non-professional employees have only recently begun to raise their pay levels through unionization and collective bargaining, also contributing to the rise in hospital costs.

In the past, hospitals have encountered problems in providing continuing education for their employees. These problems have related to the lack of supervisor training skills, unavailability of instructors and educational materials, and insufficient employees background for the material needed. One method of training, used in industry, is the management game - a training exercise which uses a model of a business situation to teach the participant some common terminology and procedures. To date, however, management games have not been developed for the specific functions found in a hospital. The purpose of this paper is to briefly outline the development and design of the Surgical Scheduler's Hospital Management Game, using the simulation language GASP II.6

\section{THE SURGICAL SCHEDULER'S GAME}

The game focuses on the scheduling function of the surgical service in a four hundred bed, short-term, non-profit, medical-surgical, teaching hospital equipped with five operating rooms. The participant is asked to assume the role of a surgical scheduler. Upon the request of a surgeon, he must determine if a surgical room is available and, if not, reach a mutualiy agreeable alternate date and time when the operation can be performed. To begin the game and to assist the participant, five data sets are provided: Data Set 1 contains the player's instructions; Data Set 2 contains a list of the operations usually performed in the hospital and the average time associated with each; Data Set 3 is the list of the surgeons who comprise the surgical staff; Data Set 4 contains the work sheets for each working day for the month of January; and Data Set 5 contains both the events to be scheduled for the first period and a Confirmation Sheet. This latter form serves as the communication device between the participant and the Game (or game administrator).

To begin play, the participant turns to Data Set 4, which contains a scheduling page for each working day of the month of January. Because this is assumed to be an on-going system, the previous surgical scheduler has already reported some operations for the days in January. The participant reviews the existing comitments and, if he wishes to make changes, he records them on the Confirmation Sheet at the end of Data Set 5. After completing the desired changes, the participant begins to work with Data Set 5 . The first event will be a scheduling request. Each such request includes the name of the surgeon making the request, the name of the operation to be performed, the patient number, the age of the patient, the date and time the surgeon would like to perform the operation, and the surgeon's estimate of how long the operation will last. The participant then decides how much time he will allow for the operation and the date and time it can be performed. He records the necessary information on the appropriate Scheduling Work Sheet and on the Confirmation Sheet. After he has completed this procedure for each schedu ing request in Data Set 5 , the participant submits his Confirmation Sheet to the game administrator. The scheduling requests are then key punched and submitted to the computer program for evaluation.

After the schedule has been processed the participant receives four reports designed to assist him in evaluating the schedule he developed. The first report is the Schedule Feedback Sheet, and it 1ists the information the participant placed on the Confirmation sheet. The second report is the Operating Room Results Report. This report provides the scheduler with the operating schedule he developed for this period and the schedule that actually resulted. The Surqical Service Performance Report, the third report, indicates in summary form how well the participant utilized the surgical facilities, how well he estimated the length of the operations and how well he met the surgeon's scheduling requests. 
The Financial Report is the final report the participant receives after each period. This latter report indicates the financial status of the surgical service. With these reports, the participant receives a new Data Set 5 and and scheduling procedure begins the second cycle.

\section{THE GASP II SIMULATION LANGUAGE}

Simulation language GASP II 6 was selected as the basic game foundation for the following reasons:

1. GASP II is FORTRAN based and requires no separate compiling system.

2. GASP II is modular and can be used on most computers which have a FORTRAN compiler.

3. Since GASP II is well documented and can easily be learned by a FORTRAN Programer.

GASP II consists of a set of FORTRAN subprograms organized to assist in performing simulation studies. It is structured to provide event control, an information storage and retrieval system, state initialization, system performance data collection, program monitoring and event reporting, statistical computation and report generation, and random variable generation.

\section{DATA COLLECTION}

One of the most important decisions to be made in the design of a management game is the quality and quantity of information that must be provided to simulate the position being assumed by the player. In order to provide realism, it was decided to further analyze data collected by Arbucklel. This data was collected from a four-hundred bed, short-term general hospital with a nine-room surgical suite. Information was collected on 4,037 operations over a seven-month period.

For purposes of the Game, it was decided that the data could be classified into operation related data, surgeon related data and patient related data. The operation related data includes the operation name file, the operation frequency distribution, the operation parameter file, the operation length distribution, the age distribution, the scheduling request distribution and the cancellation request distribution. The surgeon related data includes the surqeon name file, the surgeon operation frequency distribution and the surgeon operation matrix. The patient related data is composed of. the patient number, the date request distribution and the time request distribution. Statistical analyses were conducted, using the KolmonorovSmirnov test ${ }^{3}$ in order to determine which probability distribution was appropriate for the various types of data.

The operation name file contains the medical name for each of 320 operations. As might be expected, certain operations are performed more often than others. An operation frequency distribution file was developed containing the number of times an operation is likely to be performed. From this file an empirical probability distribution was developed to control the frequency that the operation is requested by the surgeon.

In order to simulate the length of an operation, the mean, the standard deviation, and the minimum and maximum operating time was determined for each of the 320 operations. It was determined statistically that a truncated normal distribution could represent the length of operating time required by each operation. on a particular day the number of scheduling requests was found to be Poisson distributed.

With each operation there is an associated age distribution. For example, very few persons over the age of eleven have a tonsillectomy. The mean age, the standard deviation, the minimum age, the maximum age, and the number of cases was calculated for each operation. After reviewing the actual data, it was decided to use a uniform distribution for the age, with the limits being the minimum and maximum age associated with each operation.

A study by Harris indicated a 6.4 percent cancellation rate per day. ${ }^{4}$ The analys is of Arbuckle's data indicated a 5.1 percent cancellation rate. For purposes of the game, a 5.0 percent cancellation rate was used. The number of cancellations per day was determined to be Poisson distributed.

Surgeon related data includes the surgeon name file, the surgeon operation frequency distribution and the surgeon operation matrix. A data file was developed containing a list of the fifty hypothetical surgeons who are to perform the operation. Each surgeon has a different frequency of surgical cases he performs each year. The surgeon is also limited to certain types of operations. Since this is input data, it can easily be modified to simulate the surgical staff of any hospital.

The patient and time related data and distributions are composed of the patient number, the data request distribution and the time request distribution. A patient number is assigned to each request, starting in sequence with 101. The future date on which the surgeon would like to perform the operation follows a Poisson distribution with a mean of five days and a maximum of thirty days. From Arbuckle's data it was determined that the time of day when surgeons make requests to perform an operation are uniformly distributed. For the Game operations are normally scheduled between eight o'clock and two o'clock.

Using the above distributions, data files, and parameters, it was possible to generate scheduling requests and cancellation events. From these events surgical service was developed. In each instance where a distribution or a parameter had to be specified, every attempt was made to conform to the "real world" situation. Then, as a further test, output was checked by personnel familiar with hospital operations to ascertain if any of the many dimensions of the game were arbitrary or unrealistic.

\section{DESIGN OF THE GAME}

In discrete-event simulation, the events of a system are considered at discrete points in time. These events then cause the status of a system to change. In GASP II, the time for an event to occur is either preset at the start of the simulation or set during the simulation. The Game was designed with five events.

- the Initialization Event

- the Evaluation Event

- the Scheduling Request Event

- the Cancellation Request Event

- the Termination Event 
Figure 1 is a macro flow chart of the game. The program starts with the Initialization Event by reading the operating and surgeon data. The first participant's initialization data and request decision cards are read next. The information on the decision cards is coded and checked for errors. Once the data has been prepared, the Evaluation Event starts. That day's operating schedule is simulated, measures of effectiveness are calculated, and the financial status is determined. With the printing of the appropriate reports, this period is complete. The next period is started by the Scheduling and Cancellation Request Events which generate and print the scheduling and cancellation request. The Termination Event prints the data for the next period and checks to see if this is the last participant; if not, it starts over with the reading of the next player's Initialization Cards.

In terms of subroutines, the Game proceeds in the following manner:

The game starts with the Initialization Event. The main program calls ZERO and INDA. This is shown in Figure 2. ZERO enters the numerical number zero in several non-GASP II arrays and matrics. INDA reads and stores all the data described in the "Data Collection" section. This routine also calls CPRD which develop a cumulative probability distribution function for the procedure frequency distribution. Control of the program is then turned over to GAS4P which is a GASP II subroutine. GAS4P calls DATAM which initializes all GASP II variables, arrays and matrics and controls the reading of the eight data card sets associated with GASP II.

At this point all initialization has been completed and a 11 data has been read and stored, except for the schedule the player has developed for this period. GAS4P now scans its time file with FIND, RMOVE and SET and locates the first event scheduled, which is Event 1. HRDOT, INDS, IMPRS, and DFLC read and print the schedule the player developed for this period, reads his previous schedules, codes the data, and checks for inconsistencies and errors.

The second part of Event 1 is the evaluation of the schedule developed for thi.s period. AFSCHD simulates the actual schedule and determines the best schedule that could be developed for this period. HCOST calculates the financial report and the performance report. This completes Event 1. All the statistics needed to do the calculations have been collected by GASP II subroutine COLCT.

The Scheduling Request Event is Event 2. CLRVD generates the scheduling request event, including the operation name, the surgeon making the request, the date and time the surgeon would like to perform the operation, the length of time the operation will require, the patient number and age, and when the next request will be made. GASP II handles the generation of data through process generators RNORM, UNFRM, and NPOSN; all the storage of: the information generated by the scheduling request through subroutine FILEM; and all the statistics that are collected with subroutine COLCT.

The Cancellation Event is Event 3. CLCND generates and stores the cancellation request, which determines which operation is to be cancelled and when the next cancellation request will be made. GASP II routines find where the operation to be cancelled is stored, remove it, and store it in a special location. The Scheduling Request Event and the Cancellation Event can occur in any order.
The Termination Event is Event 4. ENDSM, HCLRVD, AFORM, DATP, MONP, and HRDOT print the form and punch cards for the next period, generate scheduling requests if the total hours of requests are below a predetermined level, and print the forms and punch the cards needed for the next period.

GAS4P now returns control to MAIN which checks to see if this is the final player in the aame. If it is not, control is returned to GAS4P and the next player is processed.

The game has two unique features, the best schedule index and the adaptive learning device. An algorithm determines the surqical suite profit (ioss) that would result if the best schedule were developed. This profit (loss) is compared with the profit (loss) of the player's schedule. An algorithm, using the actual and the best profit (loss) values, is used to calculate best schedule index. This index gives the player immediate feedback by comparing his schedule with the best schedule.

The adaptive learning device causes the difficulty of the scheduling function to become more difficult by increasing the demand for service. This occurs whenever the performance of the player, measured by the best schedule index, exceeds a specified level.

\section{SUMMARY}

The primary advantage of GASP II is its flexibility. It usually is only a minor exercise to modify a routine to meet the specific requirements of the programer. This is especially true since GASP II is a FORTRAN based language and is wel1 documented. A second advantage of GASP II is the storage requirement. Since the size of all the GASP II arrays and matrics are within the control of the programmer, they can be tailored to the particular simulation being developed. The primary disadvantage of GASP II is that it requires a higher level of programing skill than some of the other simulation languages.

The Surgical Scheduler's Management Game is composed of a FORTRAN IV computer program and three instruction manuals. The Game Adminjstrator's Manual, the Player's Manual, and the Programmer's Manual contain instructions for running, playing and modifying the Game. The FORTRAN IV computer program contains 5,844 statements of which 2,768 are executable statements. The game requires a maximum storage of $130 \mathrm{~K}$ with a lower limit of 50K. Execution time on a IBM $360 / 65$ under FORTRAN IV version 17 was $1.3+0.4 *$ NT minutes where NT is the number of teams playing. The Game will print $10+10$ *NT pages and punch 60*NT cards per period. The Game has been played by more than 100 students and is being tested in a hospital surgical department. 


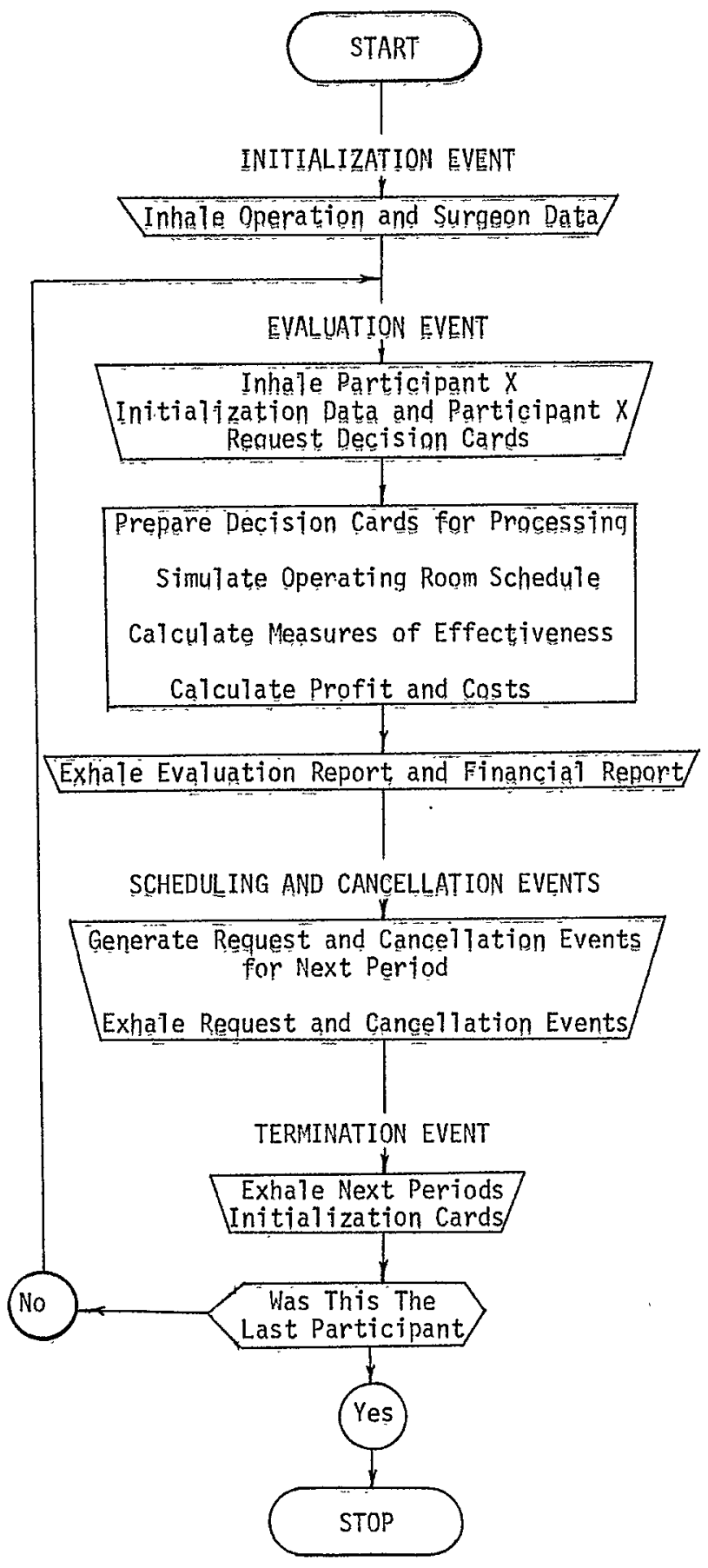

Figure 1 -- MACRO FLOW CHART

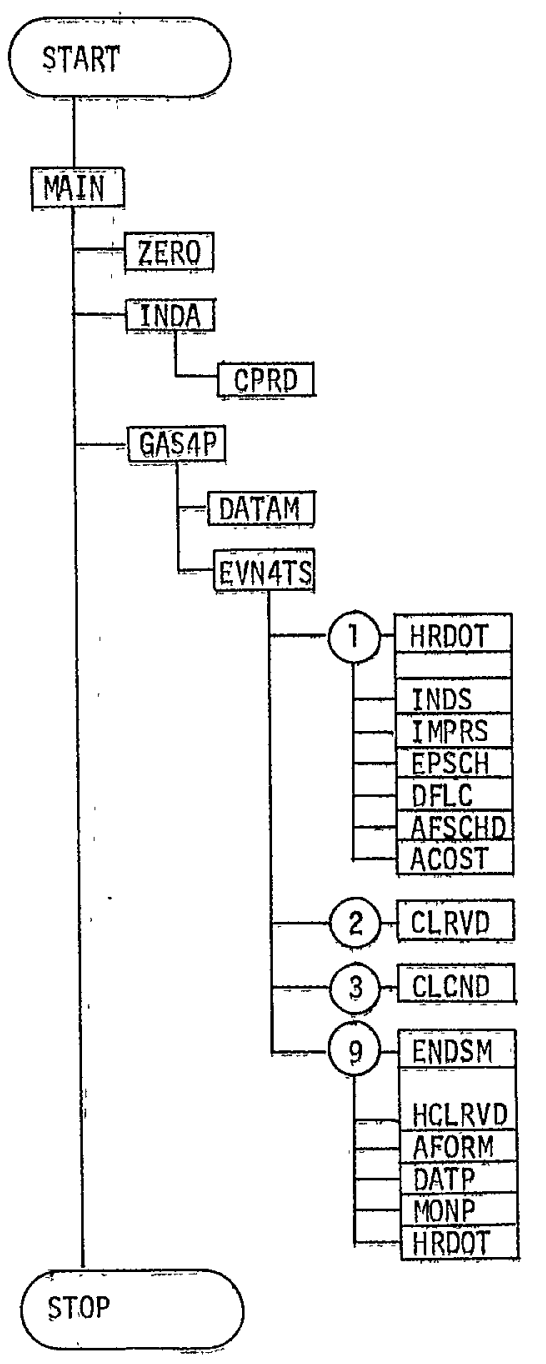

Figure?

PRIMARY SUBPROGRAM FLOW CHART 
1. Arbuckle, James Albert. Analys is of a Surgical Operating Room Scheduling System. Unpublished M.S. Thesis. BTacksburg, Virainia, Library, Virginia Polytechnic Institute. 1970.

2. Business Week. "The \$60-Billion Crisis Over Medical Care." Reprint. New York, New York, McGraw-Hi11, Inc. January, 1970.

3. Dineen, Robert A. A FORTRAN Based Method for Fitting Frequency Curves. Master's Thesis, Arizona State University, June, 1969.

4. Harris, David H. Surgical Suite Analysis of Butterworth Hospital, Grand Rapids, Michigan. Ann Arbor, Michigan, Community Systems Foundation. November, 1965.

5. Kibbee, Joel M., Craft, Clifford J., and Nanus, Burt. Management Games, A New Technique for Executive Development. New York, New York, Reinhold Publishing Corporation. 1961.

6. Pritsker, A, Alan and Kiviat, Philip J. Simulation with GASP II. Englewood Cliffs, New Jersey, Prentice-HaT1, Inc. 1969.

7. Schmidt, J. William and Taylor, Robert E. Simulation and Analysis of Industrial systems. Homewood, Illinois, Richard D. Irwin, Inc., 1970.

8. Torgersen, Paul E. and Taylor, Robert E. The Urban Mass Transit Game. Technical Report Phase T-B. Blacksburg, Virginia, Virginia Polytechnic Institute. 1969. 